\section{Relations of diet and physical activity to bone mass and height in black and white adolescents}

\author{
Bernard Gutin, 1 \\ Inger S. Stallmann-Jorgensen, 1 \\ Anh H. Le, 2 Maribeth H. Johnson, 3 \\ Yanbin Dong1 \\ 1 Georgia Prevention Institute, Georgia \\ Health Sciences University, Augusta, \\ Georgia; 2Department of Biostatistics, \\ University of Alabama at Birmingham, \\ Birmingham, Alabama; ${ }^{3}$ Department \\ of Biostatistics, Georgia Health Sciences \\ University, Augusta, Georgia, USA
}

\section{Abstract}

Because the development of healthy bodies during the years of growth has life-long health consequences, it is important to understand the early influences of diet and physical activity (PA). One way to generate hypotheses concerning such influences is to conduct cross-sectional studies of how diet and PA are related to different components of body composition. The subjects were 660 black and white adolescents. Total body bone mineral content (BMC) was measured with dual-energy X-ray absorptiometry; free-living diet and PA were assessed with 4-7 separate 24 -h recalls. The main dietary variables investigated were: total energy intake, macronutrient distribution (\%), dairy servings, vitamin $\mathrm{D}$, and calcium. The main PA variables were hours of moderate PA (3-6 METs) and vigorous PA ( $>6$ METs). BMC was higher in blacks than in whites $(\mathrm{P}<0.01)$ and it increased more in boys than in girls (age by sex interaction) as age increased $(\mathrm{P}<0.01)$. After adjustment for age, race and sex, higher levels of BMC were associated with higher levels of energy intake, dairy servings, calcium, vitamin $\mathrm{D}$, and vigorous PA (all P 's<0.05). In the multivariable model, significant and independent proportions of the variance in $\mathrm{BMC}$ were explained by race, the age by sex interaction, calcium, and vigorous $\mathrm{PA}$ (all $P$ 's $<0.01$ ). When height was used as the outcome variable, similar diet results were obtained; however, there was a sex by vigorous PA interaction, such that vigorous PA was associated with height only in the girls. These data are consistent with the hypothesis that the bone mass and height of growing youths are positively influenced by higher dietary intake of energy and dairy foods, along with sufficient amounts of vigorous PA. This hypothesis needs to be tested in randomized controlled trials.

\section{Introduction}

Body composition develops during the early years of life and tends to track into later years. ${ }^{1}$ Therefore, understanding the effects of lifestyle factors such as diet and physical activity (PA) can guide the implementation of social policies and interventions that can help youths to develop healthy bodies. One way to gain insight into these influences is to conduct cross-sectional investigations in order to generate hypotheses concerning how diet and PA influence body composition; these hypotheses can then be tested in randomized controlled trials to arrive at causal conclusions.

The current paper is based on data collected in a project conducted at the Georgia Health Sciences University (formerly the Medical College of Georgia) entitled Lifestyle, adiposity and cardiovascular health of youths. Earlier publications from this project found that adolescents who engage in more vigorous PA and ingest more dietary energy than their agemates tend to have relatively low levels of body fatness, ${ }^{2}$ thus putting them at lower risk for cardiometabolic diseases. ${ }^{3}$ In a similar manner, prevention of osteoporosis probably begins in childhood. Thus, it is important to determine if the relations of diet and PA to lower adiposity levels are mirrored by their relations to higher bone mass.

The skeletal benefits of physical activity in adolescents are maintained into young adulthood and youths who develop high levels of bone mass early in life are less likely to decline to osteoporotic levels later in life. ${ }^{4}$ It has been shown retrospectively that women who had the highest levels of bone mass in their postmenopausal years had engaged in the most PA during their teen years. ${ }^{5}$

There is evidence that blacks and boys have higher levels of bone mass than whites and girls, respectively. ${ }^{6}$ Moreover, there is evidence that higher levels of bone mass are associated with dietary factors such as intake of dairy foods, ${ }^{7}$ along with weight-bearing PA. ${ }^{5}$ However, little is known about the interactions of diet and PA with age, race or sex, and it is unclear if bone mass is more closely associated with vigorous PA than with moderate PA. Because bone mineral content (BMC) is the index of bone mass that is most clearly related to bone strength in growing youths, ${ }^{8}$ the primary purpose of this study was to determine the relations and potential interactions of freeliving diet and PA to BMC in a relatively large bi-racial sample of 660 boys and girls.

A secondary purpose of this study was to explore the relations of diet and PA to standing height, which is potentially influenced by diet and PA in ways that are similar to how diet and PA influence bone mass. For example, one study found that children who avoided drink-
Correspondence: Bernard Gutin, 10161 Mizner Lane, Raleigh, NC 27617, USA

Tel. 919.425.1300 - Fax. 706.721.7150.

E-mail: bernardgutin@yahoo.com

Key words: bone mass, youths, diet, physical activity.

Acknowledgment: this study was supported by the National Institutes of Health (HL64157).

Contributions: BG primary funding acquisition, study design and manuscript writing; IS data collection and manuscript writing; $\mathrm{AL}, \mathrm{MJ}$, data analysis and tables and figures preparation; YD, funding acquisition assistance and manuscript preparation supervision.

Conflict of interest: the authors report no conflicts of interest.

This research was carried out at the Georgia Prevention Institute, Department of Pediatrics, Georgia Health Sciences University (formerly Medical College of Georgia).

Received for publication: 18 January 2011. Accepted for publication: 24 March 2011.

This work is licensed under a Creative Commons Attribution 3.0 License (by-nc 3.0).

(C) Copyright B. Gutin et al., 2011

Licensee PAGEPress, Italy

Pediatric Reports 2011; 3:e10

doi:10.4081/pr.2011.e10

ing milk had relatively low BMC and were relatively short, ${ }^{9}$ another found that vitamin D insufficiency was associated with decreased height, ${ }^{10}$ and secular changes in growth of children are often attributed to nutritional factors. ${ }^{11}$ Randomized controlled trials have shown that vigorous PA has a positive influence on bone mass. ${ }^{12}$ However, little is known about whether normal variations in diet and PA within one country have a measurable influence on linear growth, or whether diet and PA interact with each other, or with race and sex, in their relations to bone mass and growth.

A lifestyle that includes large amounts of PA can allow youths to ingest relatively large amounts of dietary energy without accumulating excessive amounts of fat mass. ${ }^{2}$ Moreover, a diet high in energy may allow youths to ingest sufficient amounts of bone- and growth-enhancing nutrients. Therefore, we hypothesized that higher levels of BMC and height would be related to daily hours of moderate and vigorous PA, daily dairy servings, and dietary intake of energy, vitamin D and calcium. 


\section{Materials and Methods}

\section{Subjects}

Youths aged 14-18 were recruited from high schools in the Augusta, Georgia area. Schools that enrolled both black and white students were selected. Because there were very few Asians or Hispanics in Augusta, they were not recruited for this study. With approval from superintendents and school principals, flyers were distributed to all students in the schools. Interested students who responded to the flyers and called the institute to participate were screened over the telephone to determine preliminary eligibility. Criteria included: being 14-18 years of age; being of the black or white race by selfdesignation; being enrolled in school; having a body weight $\leq 300 \mathrm{lbs}$ (weight limit for the dual-energy $\mathrm{x}$-ray absorptiometer-DXA); being of good health and females being nonpregnant; using no medications that could influence study results including those for birth control, attention-deficit-disorders, and asthma; and not having contraindications to completing any of the testing procedures. Youths who passed the telephone screening were invited to the Georgia Prevention Institute accompanied by their parents if they were minors, to learn about the study. All subjects and the parents of subjects who were minors provided written informed assent and consent in accordance with requirements of our Human Assurance Committee (the internal review board at the Georgia Health Sciences University) prior to study participation; this committee also approved all study procedures.

\section{Anthropometrics and body com- position}

Weight $(\mathrm{kg})$ was measured twice to the nearest $0.1 \mathrm{~kg}$ using a leveled platform scale, calibrated monthly, with the subject not wearing shoes and in light clothing (T-shirt and shorts).

Height (m) was measured twice to the nearest $0.1 \mathrm{~cm}$ using a wall-mounted stadiometer with subject not wearing shoes, standing straight against the wall with head, buttocks, and heels touching the wall, arms hanging freely at the sides and chin parallel to the floor, and after inhaling and exhaling a deep breath of air. Means of the weight and height measurements were used to derive body mass index (BMI [weight.height ${ }^{2}$ ]). DXA (Hologic QDR-4500W, software version 6.0, Waltham, MA, USA) was used to derive a 3-compartment model of body composition: fat mass, fat-free soft tissue and BMC. The intraclass correlation coefficient for repeated measurements of BMC was $>0.99 .12$
Diet and physical activity assessment

Diet and PA were measured with non-consecutive, 24 -h recalls covering the period from midnight to midnight for the previous day. The first two recall interviews were conducted in person at our institute, with the rest conducted by phone weekly and completed within 12 weeks. Subjects were blinded to the telephone recall schedule to minimize the potential for under-eating during the time frame for the 24 $\mathrm{h}$ recall and calls were made on all days of the week to obtain diet data representing both weekdays and weekend days. The diet recall data was captured using the Nutrition Data System for Research (NDS-R) ${ }^{14}$ which utilizes a multiple pass, computer-assisted interview approach. To minimize subject fatigue with the recall process, which might reduce reliability, interviewers were trained to conduct the recalls in $30 \mathrm{~min}$ or less. Subjects were not interviewed about days when they had been ill, or days that fell on a major holiday. Youths who provided at least four recalls were included in the analyses. Fifty-two percent of the youths had recalls for all 7 days. The dietary and PA recalls were conducted concurrently by a trained dietitian or dietetic intern.

The NDS-R Food and Nutrient Database groups food into nine major food categories: fruits, vegetables, grains, sweets, miscellaneous, fats, beverages, meats, and dairy/ nondairy alternatives. Serving sizes were based on the Dietary Guidelines for Americans $2005,{ }^{15}$ or on Food and Drug Administration label serving sizes. We examined total energy intake and percentage of energy from carbohydrates (\% $\mathrm{CHO}$ ), protein (\%PRO), and fat (\%FAT), as well as dietary vitamin $\mathrm{D}(\mu \mathrm{g})$ and calcium $(\mathrm{mg})$.

For the PA assessment, we compiled a list of activities typical for adolescents, and assigned metabolic equivalent values (METs) to the activities based on the compendium of physical activities. ${ }^{16}$ Self-reported PA was quantified using our version of a validated previous day PA recall (PAR) instrument, recording activities in 48, 30-min time blocks, by extending the time frame from covering only the after school hours of 3:00 p.m. to 11:30 p.m. to covering the full 24-h day. ${ }^{17}$ Subjects were asked to state their level of effort (light, moderate, or vigorous) for each activity. Activities were re-categorized based on a combination of the activity and the level of effort; e.g. light running was assigned a higher metabolic equivalent (MET) level than light walking. In the final categorization, light PA was $<3$ METs, moderate PA was 3-6 METs and vigorous PA was $>6$ METs. In general, moderate PA involves activities such as brisk walking while vigorous PA involves activities that involve running. These cutpoints are somewhat arbitrary; if the overall project focused only on bone, brisk walking might be characterized as a light activity rather than a moderate activity, running might be a moderate activity and activities such as jumping might be classified as vigorous. However, the parent project focused on visceral and general adiposity as well as bone. Therefore, we used terminology and metabolic breakpoints that had been previously used with this PA measurement instrument.

\section{Statistical analyses}

The SAS program version 9.1.3 (SAS Institute, Inc, Cary, NC, USA) was used for statistical analyses with the level of significance set at 0.05 . Variables were checked for normality and appropriate transformations were applied if necessary. Descriptive race and sex comparisons were made using analysis of covariance, adjusting for age. However, detailed consideration of the demographic differences in diet and PA is beyond the scope of this paper; our focus was on whether the associations of diet and PA with BMC could be generalized across race and sex.

Pearson correlations were used to identify associations among variables and to identify possible multicollinearity issues that could arise in model building. A base model containing the demographic variables of age, sex, and race was first established to describe the effects of these demographic variables on the outcome variables of BMC and height. We considered using height as part of the base model to control for size, but it seemed possible that the diet and PA variables might be associated with height in the same way that they were associated with BMC, in which case inclusion of height in the base model would obscure the relations of diet and PA to BMC. Adjusting for height would also be more important if the project were focused on sex differences in BMC. However, this paper does not focus on sex differences but rather controls for sex to see if the relations of diet and PA to BMC and height can be generalized to both sexes.

Then we added each diet and PA predictor variable to the base model, one at a time, in order to determine whether each one explained a significant additional proportion of the variance in the outcome. In these analyses, we also examined possible interactions between the demographic and predictor variables. Then a full multivariate model was obtained by combining diet and PA variables that had explained significant proportions of the BMC variance. We also examined possible interactions between predictor variables for the full model. Similar statistical techniques were applied to the analyses that used height as the outcome variable. 


\section{Results}

Descriptive data and the influences of age, race, and sex are shown in Table 1 . Weight, height, BMC, and moderate PA increased with age. Boys had higher BMC than girls and blacks had higher BMC than whites. For moderate PA, males had higher levels than females. For vigorous PA, there was a race by sex interaction, such that black males had the highest levels while black females had the lowest levels. With respect to diet variables, whites had higher intake of energy, \% $\mathrm{CHO}$, \%PRO, calcium, vitamin D and dairy servings than blacks. Males also had higher intake of all these dietary factors, except for \% $\mathrm{CHO}$, than females. Blacks had higher intake of \%FAT than whites.

Table 2 shows the results for the base model of age, race and sex. As age increased, boys increased more than girls in BMC. There was no significant race by sex interaction. Table 2 also shows the regression results in which each diet and PA variable was added to the base model, one at a time. With respect to diet, significant additional proportions of the variance in BMC were explained by intake of energy, calcium, vitamin D, and dairy servings. With respect to PA, a significant proportion of the BMC variance was explained by vigorous PA, but not moderate PA.

When height was used as the outcome measurement, all the same dietary variables were related to height (all $\mathrm{P}^{\prime} \mathrm{s}<0.05$, data not shown). For PA, there was a significant interaction between sex and vigorous $P A$ $(\mathrm{P}=0.014)$, such that vigorous $\mathrm{PA}$ was positively associated with height in girls, but not in boys.

In building a multivariate model of diet and PA, we considered the interrelationships among variables to avoid multicollinearity. The four significant diet variables were intercorrelated ( $\mathrm{r}=0.50$ to 0.91 ), and the relationships with BMC and height were similar. In the interests of parsimony, we selected one main dietary variable to use in the multivariable model. Table 3 shows the full model results for BMC. Both calcium intake and vigorous PA explained independent proportions of the variance in BMC, and neither interacted with age, race, sex or each other. This indicates that the influence of diet and PA could be generalized across the demographic groups.

When height was used as the outcome variable, the multivariate model showed that calcium and the interaction between sex and vigorous PA were significant $\left(\mathrm{P}^{\prime} \mathrm{s}<0.05\right)$; vigorous PA explained a significant proportion of the variance in the girls, but not in the boys and the proportion of the variance explained was 0.02 .
Table 1. Descriptive characteristics for subjects in the study. ${ }^{1}$

\begin{tabular}{|c|c|c|c|c|c|}
\hline Variable & $\begin{array}{c}\text { White males } \\
(\mathrm{n}=168)\end{array}$ & $\begin{array}{l}\text { White females } \\
\qquad(\mathrm{n}=170)\end{array}$ & $\begin{array}{l}\text { Black males } \\
\quad(n=163)\end{array}$ & $\begin{array}{l}\text { Black females } \\
(\mathrm{n}=159)\end{array}$ & $\begin{array}{c}\text { Group } \\
\text { comparison }^{2}\end{array}$ \\
\hline Age (years) & $16.2(1.2)$ & $16.0(1.1)$ & $16.0(1.2)$ & $16.3(1.2)$ & $\mathrm{R}^{*} \mathrm{~S}$ \\
\hline Height (cm) & $174.4(7.3)$ & $163.2(5.4)$ & $173.8(8.0)$ & $162.7(6.1)$ & $\uparrow \mathrm{w} /$ Age, $\mathrm{M}>\mathrm{F}$ \\
\hline Weight (kg) & $68.6(14.6)$ & $58.4(12.0)$ & $70.8(17.2)$ & $66.5(17.7)$ & $\uparrow \mathrm{w} / \mathrm{Age}, \mathrm{R}^{*} \mathrm{~S}$ \\
\hline BMI $\left(\mathrm{kg} \cdot \mathrm{m}^{-2}\right)$ & $22.5(4.3)$ & $21.9(4.1)$ & $23.4(5.2)$ & $25.0(6.2)$ & $\mathrm{R}^{*} \mathrm{~S}$ \\
\hline $\operatorname{BMD}\left(\mathrm{g} \cdot \mathrm{m}^{-2}\right)$ & $1.08(0.11)$ & $1.04(0.08)$ & $1.15(0.13)$ & $1.13(0.09) \uparrow$ & $\uparrow$ w/Age, $M>F, B>W$ \\
\hline $\mathrm{BMC}(\mathrm{g})$ & 2328 (434) & 1946 (247) & 2505 (498) & $2230(344) \uparrow$ & $\uparrow$ w/Age, $M>F, B>W$ \\
\hline Body fat (\%) & $19.2(8.34)$ & $29.2(7.18)$ & $17.3(9.11)$ & 30.5 (8.47) & $\mathrm{R} * \mathrm{~S}$ \\
\hline $\begin{array}{l}\text { Moderate PA } \\
\text { (hours per day) }\end{array}$ & $1.27(1.04)$ & $1.07(0.75)$ & $1.12(0.790)$ & $1.06(0.83)$ & $\uparrow \mathrm{w} /$ Age, $\mathrm{M}>\mathrm{F}$ \\
\hline Vigorous PA & $0.78(0.690)$ & $0.43(0.48)$ & $0.95(0.72)$ & $0.27(0.72)$ & $\mathrm{R} * \mathrm{~S}$ \\
\hline
\end{tabular}

(hours per day)

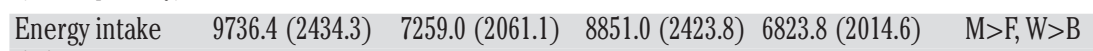

(kJ)

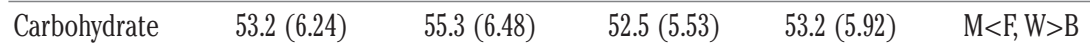

intake (\%)

\begin{tabular}{lccccc} 
Protein intake (\%) & $14.6(2.84)$ & $13.6(2.76)$ & $13.8(2.13)$ & $13.5(2.76)$ & M >F, W>B \\
\hline Fat intake (\%) & $32.9(4.79)$ & $32.4(5.12)$ & $34.5(4.17)$ & $34.2(4.46)$ & B >W \\
$\begin{array}{lcccc}\text { Calcium } \\
\text { intake (mg) }\end{array}$ & $982.1(423.4)$ & $731.7(321.9)$ & $725.6(263.3)$ & $524.3(217.4)$ & M >F, W>B \\
$\begin{array}{l}\text { Vitamin D } \\
\text { intake }(\mu g)\end{array}$ & $5.37(3.42)$ & $3.74(2.51)$ & $3.95(2.14)$ & $2.76(1.60)$ & M >F, W>B
\end{tabular}

intake $(\mu g)$

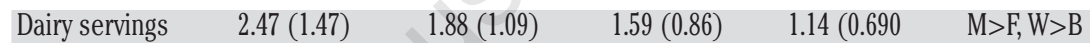

${ }^{1}$ All values are means (SD). ${ }^{2}$ Group comparisons were conducted by analyses of covariance (sex by race) after adjustment for age $(\mathrm{P}<0.05)$. $R^{*} S$, race by sex interaction; $\uparrow$ w/Age, the variable increases as age increases; M, males; F, females; BMD, bone mineral density; $W$, whites; $B$, blacks; BMC, bone mineral content; PA, physical activity; kJ, kilojoules

Table 2. Unstandardized regression coefficients $(\beta)$, SEs, and model $\mathbf{R}^{2}$ examining the association of physical activity and dietary factors with bone mineral content after adjustment for base model factors from multiple regression.

\begin{tabular}{lccccc}
\hline Model & Predictor variable & $\beta$ & SE & P & $\mathbb{R}^{2}$ \\
1 & Base model & - & - & - & 0.343 \\
2 & Moderate PA & 8.4 & 16 & 0.61 & 0.344 \\
\hline 3 & Vigorous PA & 66 & 23 & 0.004 & 0.352 \\
4 & Calcium & 0.16 & 0.04 & 0.0002 & 0.357 \\
\hline 5 & Vitamin D & 13 & 5.5 & 0.020 & 0.349 \\
6 & Dairy servings & 41 & 13 & 0.002 & 0.353 \\
\hline 7 & Energy intake & 0.06 & 0.03 & 0.024 & 0.348 \\
8 & Protein intake (\%) & 9.7 & 5.3 & 0.14 & 0.345 \\
\hline 9 & Carbohydrate intake (\%) & -2.7 & 2.3 & 0.24 & 0.345 \\
10 & Fat intake (\%) & 1.5 & 3.0 & 0.60 & 0.344 \\
\hline
\end{tabular}

The base model contains age, race, sex, and the age by sex interaction. Models 2-10 represent the addition of the predictor variable to the base model. In all models there was a significant race effect $(\mathrm{W}<\mathrm{B})$ and age by sex interaction (M increase more than $\mathrm{F}$ across age) $(\mathrm{P}<0.05)$. BMC, bone mineral content; PA, physical activity.

Table 3. Unstandardized regression coefficients $(\beta)$, SEs, and model $\mathbf{R}^{2}$ examining the simultaneous association of vigorous physical activity and calcium intake with bone mineral contentfrom multiple regression.

\begin{tabular}{lcc} 
& $\beta($ SE) & $P$ \\
Intercept & $1448(274)$ & - \\
Race $^{1}$ & $-279(29)$ & $<0.0001$ \\
\hline Sex $^{2}$ & $-2061(378)$ & $<0.0001$ \\
Age & $41(17)$ & $<0.0001$ \\
\hline Age*Sex & $145(23)$ & $<0.0001$ \\
Calcium intake $^{2}$ & $0.15(0.04)$ & 0.0004 \\
\hline Vigorous PA & $58(23)$ & 0.0097 \\
Model R2 & 0.364 & -
\end{tabular}

White race is reference group; 2male sex is reference group; PA, physical activity. 
The joint effects of calcium and vigorous PA on BMC are illustrated in Figure 1. Although the statistical analyses were done on continuous variables, for purposes of illustration in the figure, the sample was broken down into groups. For vigorous PA, the total sample was broken down into three groups: i) those who reported no vigorous $\mathrm{PA}$ at all; ii) those who reported some, but $<1$ hour/day; and iii) those who reported 1 hour or more per day. For calcium intake, the total sample was divided into thirds. It is clear that the highest BMC was found in those youths who ingested the most calcium and did the most vigorous PA.

\section{Discussion}

This study found that the bone mass and height of adolescents were associated with diet and PA. The main dietary factors were energy intake and those embodied in dairy products (i.e., calcium and vitamin D). With respect to $\mathrm{PA}$, vigorous $\mathrm{PA}$ rather than moderate PA was associated with BMC. Vigorous PA includes activities that place mechanical strain on the musculo-skeletal system, such as running, basketball, soccer and dance. These results are consistent with the hypothesis that the ingestion of dairy products and associated nutrients provide the substrate for bone accretion while vigorous PA provides the mechanical stimulation needed to promote bone formation. The dairy result is consistent with those of other investigators. ${ }^{7,18}$ It is noteworthy that the diet and PA factors explained independent proportions of the variance in BMC, whereas Rowlands et al.,19 found a positive interaction between calcium intake and PA in 8-11 year old children. Perhaps such an interaction is more likely to occur in younger children because their levels of moderate-vigorous PA are so much higher than those of teenagers. ${ }^{20,21}$

The importance of vigorous PA to optimal development of bone and fat-free mass is consistent with the results of projects that have investigated the relationship of physical fitness to $\mathrm{BMC}^{22}$ as well as intervention projects that have employed high-intensity exercise. ${ }^{13}$ Animal studies have also emphasized the importance of relatively high-intensity, weightbearing exercise to provide optimal mechanical load to the skeleton. ${ }^{23}$ Furthermore, increased lean mass is associated with even greater gains in bone density and bone strength when occurring in conjunction with high intensity PA. ${ }^{24}$

It is noteworthy that vigorous $\mathrm{PA}$, rather than moderate PA, is also associated with lower fatness levels in youths. ${ }^{25}$ Again, these findings are consistent with results from animal studies in which increased muscle mass and PA are associated with decreased body fat and resistance to body fat gain. ${ }^{26}$ This study complements another study of this adolescent cohort ${ }^{2}$ by showing that energy intake and vigorous PA were related not only to higher BMC, but also to low levels of percent body fat. Thus, the development of desirable body composition seems to depend on high levels of energy intake and associated nutrients, in combination with adequate amounts of vigorous PA. ${ }^{27}$ The energy and nutrients provide the substrate for optimal body composition development while the vigorous PA encourages partition of the ingested nutrients into bone and muscle rather than fat. This is consistent with recent findings that mechanical stimulation alters stem cell proliferation and differentiation in the direction of greater osteogenesis and lesser adipogenesis. ${ }^{28}$ It appears that only vigorous PA provides sufficient mechanical stimulation to the tissues to influence stem cells to differentiate into lean tissue (bone and muscle) rather than fat.

We found that the dietary factors associated with BMC were similar to those associated with height, which is consistent with the idea that improved nutrition, including dairy products, can have a favorable influence on growth. ${ }^{29}$ However, we found that the models for BMC and height were somewhat different, in that vigorous PA was associated with height only in the girls. Perhaps the relatively low average levels of vigorous PA in the girls led the impact of the higher levels in some girls to have a greater influence on growth. However, it has also been suggested that weight-bearing bones are more sensitive to physical exercise in boys than in girls during pre-and early puberty. ${ }^{30}$

For BMC, we found an interaction between age and sex, such that BMC increased more during the adolescent years in the boys than in the girls; this is consistent with the malefemale divergence in fat-free mass during puberty, which may be subserved by differences in sex hormones. ${ }^{31}$

It is noteworthy that the effects of diet and vigorous PA did not interact with race and sex. Thus, lifestyle recommendations can be similar for black and white youths of both sexes.

In light of the roles that vigorous PA and dairy intake play in helping youths to develop healthy bodies, it is worrisome that in the USA moderate-vigorous PA declines markedly during the adolescent years, ${ }^{20,21}$ and that only $\sim 15 \%$ of the youths in our sample met the recommended level of three dairy servings per day suggested by the 2005 Dietary Guidelines for Americans. ${ }^{15}$ Our results are consistent with data from the 1999-2004 NHANES which showed that American adolescents tend to be deficient in dairy intake. ${ }^{32}$

This study had some limitations. One concerns the fact that the key outcome variable was BMC, which is only an index of bone mass

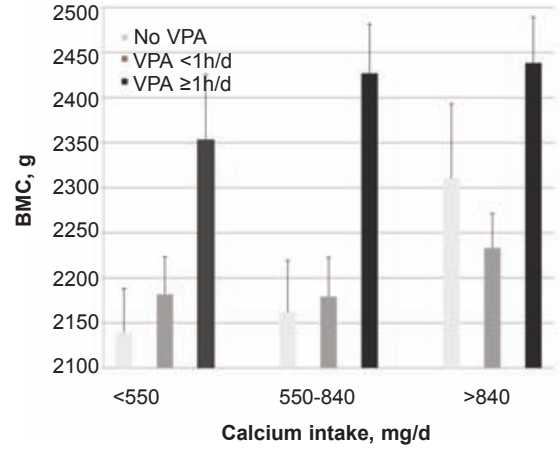

Figure 1. Illustration of the joint relations of vigorous $\mathrm{PA}$ and calcium intake to bone mineral content. BMC (SE), adjusted for age, race and sex, in relation to categories of calcium intake and vigorous PA. Although the statistical analyses were done on continuous variables, the figure breaks the calcium and vigorous PA variables into groups for purposes of illustration. For calcium intake, the youths were broken down into thirds; thus, there were 220 in each category. The number of youths in each of the vigorous PA categories is given in the legend. Both calcium intake and vigorous $P A$ explained significant independent proportions of the variance in $\mathrm{BMC}(\mathrm{P}<\mathbf{0 . 0 1})$ beyond the variance explained by age, race and sex (see Table 3). BMC, bone mineral content; PA, physical activity.

and does not provide information about bone architecture, which also contributes to bone strength. ${ }^{33}$ Another limitation was our reliance on recall of diet and PA rather than more objective measurements. Nonetheless, the recall procedures we used to measure free-living diet and PA were more extensive than have been commonly used in large-scale epidemiologic studies, in that we conducted seven independent 24-hr recalls; this provided information about all days of the week and did not require that the youths remember more than one day of diet and PA each time. Our use of a PA assessment that was based on metabolic equivalents rather than bone-specific variables is an important limitation. This reflected the fact that the data for this paper were collected as part of a larger project that was designed to see how diet and PA are related to visceral and general adiposity, ${ }^{2}$ and how adiposity is related to cardiometabolic biomarkers. ${ }^{3}$ Our findings that vigorous $\mathrm{PA}$, but not moderate $\mathrm{PA}$, is related to higher bone mass is complementary to those findings.

Another limitation involves our use of only dietary vitamin $\mathrm{D}$ rather than plasma levels of vitamin $\mathrm{D}$, which is influenced by exposure to sunlight as well as diet. ${ }^{34}$

The most important limitation is the crosssectional nature of the investigation, which makes it necessary to be tentative in drawing causal conclusions. For example, a hereditary predisposition to be tall and have greater bone 
mass may have led some youths, especially the girls, to engage in vigorous sports such as basketball. The greater metabolic rate of the taller youths might then stimulate them to eat more, including more dairy products. Of course, it is then possible that the greater amount of vigorous PA and energy/nutrient intake might contribute to further accretion of bone mass. Indeed, such a circular pattern is quite likely. Prospective cohort designs allow observation of the relations over time. In this regard, our results are consistent with two recent reports. In a 7-year follow-up study in girls, bone mass accrual was associated with a combined diet index that included several of the variables we found to be significantly related to BMC; however, these investigators did not find bone mass to be associated with a general index of leisure-time PA. ${ }^{1}$ Another study ${ }^{35}$ found that walking or cycling to school (ie, moderate PA) was not associated with greater accrual of bone mass over a 2 -year period. These results show the importance of distinguishing between different intensities of PA, which allowed us to show that vigorous PA, but not moderate PA, was associated with BMC. The long-term implications of enhanced bone mass in adolescence is illustrated by a prospective cohort study that followed the subjects into young adulthood and showed that the skeletal benefits of physical activity in adolescents were maintained. ${ }^{4}$ From a public health perspective, we need to carry these findings forward by seeing what happens when diet and $\mathrm{PA}$ are controlled in a prospective manner, using experimental designs.

In conclusion, in black and white adolescents the main dietary variables related to bone mass and height were energy intake, calcium, vitamin D and servings of dairy products. Because these variables were interrelated, it was not possible to determine which one or combination of these was actually responsible for the relation to BMC. With respect to PA, $\mathrm{BMC}$ was related to vigorous $\mathrm{PA}$, rather than to moderate PA. Taken together with recent evidence showing that lower levels of body fatness are also associated with higher levels of energy intake and vigorous PA, these results suggest that interventions designed to help youths develop healthy bodies should emphasize the positive message of engagement in vigorous $\mathrm{PA}$ and ingestion of sufficient energy and nutrients, rather than the negative message of energy intake restriction which might be contrary to the biologic needs of healthy growth. ${ }^{36}$

\section{References}

1. Cheng S, Völgyi E, Tylavsky F et al. Traitspecific tracking and determinants of body composition: a 7-year follow-up study of pubertal growth in girls. BMC Med 2009;7:5.

2. Stallmann-Jorgensen I, Gutin B, HatfieldLaube J et al. General and visceral adiposity in black and white adolescents and their relation with reported physical activity and diet. Int J Obes 2007;31:622-9.

3. Gutin B, Johnson M, Humphries M et al. Relationship of visceral adiposity to cardiovascular disease risk factors in black and white teens. Obesity 2007;15:1029-35.

4. Baxter-Jones A, Kontulainen S, Faulkner R et al. A longitudinal study of the relationship of physical activity to bone mineral accrual from adolescence to young adulthood. Bone 2008;43:1101-7.

5. Rideout C, McKay H, Barr S. Self-reported lifetime physical activity and areal bone mineral density in healthy postmenopausal women: the importance of teenage activity. Calcif Tissue Int 2006; 79:214-22.

6. Walker M, Novotny R, Bilezikian J et al. Race and diet interactions in the acquisition, maintenance, and loss of bone. J Nutr 2008;138:1256S-60S.

7. Moore L, Bradlee M, Gao D et al. Effects of average childhood dairy intake on adolescent bone health. J Pediatr 2008;153:66773.

8. Heaney R. BMD: the problem. Osteoporos Int 2005;16:1013-15.

9. Black R, Williams S, Jones I et al. Children who avoid drinking cow milk have low dietary calcium intakes and poor bone health. Am J Clin Nutr 2002;76:675-80.

10. Kremer R, Campbell P, Reinhardt T et al. Vitamin D status and its relationship to body fat, final height, and peak bone mass in young women. J Clin Endocrinol Metab 2009;94:67-73.

11. Hoppa R, Garlie T. Secular changes in the growth of Toronto children during the last century. Ann Human Biol 1998;25:553-61.

12. Barbeau P, Johnson M, Howe $\mathrm{C}$ et al. Ten months of exercise improves general and visceral adiposity, bone, and fitness in black girls. Obesity 2007;15:2077-85.

13. Litaker M, Barbeau P, Humphries M et al. Comparison of Hologic QDR-1000/W and 4500W DXA scanners in 13- to 18-year olds. Obes Res 2003;11:1545-52.

14. Schakel S, Sievert Y, Buzzard I. Sources of data for developing and maintaining a nutrient database. J Am Diet Assoc 1988;88:1268-71.

15. Department of Health and Human Services Web site. Washington (DC): Dietary Guidelines for Americans, 2005. Available from: http://www.health.gov/ DietaryGuidelines/.

16. Ainsworth B, Haskell W, Leon A et al. Compendium of physical activities: classification of energy costs of human physical activities. Med Sci Sports Exerc 1993;25:71-80.

17. Weston A, Petosa R, Pate R. Validation of an instrument for measurement of physical activity in youth. Med Sci Sports Exerc 1997;29:138-43.

18. Huncharek M, Muscat J, Kupelnick B. Impact of dairy products and dietary calcium on bone-mineral content in children: results of a meta-analysis. Bone 2008; 43:312-21.

19. Rowlands A, Ingledew D, Powell S et al. Interactive effects of habitual physical activity and calcium intake on bone density in boys and girls. J Appl Physiol 2004; 97:1203-8.

20. Nader P, Bradley R, Houts $R$ et al. Moderate-to-vigorous physical activity from ages 9 to 15 years. JAMA 2008;300:295-305.

21. Troiano R, Berrigan D, Dodd $\mathrm{K}$ et al. Physical activity in the United States measured by accelerometer. Med Sci Sports Exerc 2008;40:181-8.

22. Vicente-Rodriguez G, Urzanqui A, Mesana MI et al. Physical fitness effect on bone mass is mediated by the independent association between lean mass and bone mass through adolescence: a cross-sectional study. J Bone Miner Metab 2008;26:288-94.

23. Burr D, Robling A, Turner C. Effects of biomechanical stress on bones in animals. Bone 2002;30:781-6.

24. Hamrick M, Samaddar T, Pennington C et al. Increased muscle mass with myostatin deficiency improves gains in bone strength with exercise. J Bone Miner Res 2006;21:477-83.

25. Gutin B, Yin Z, Humphries $M$ et al. Relations of moderate and vigorous physical activity to fitness and fatness in adolescents. Am J Clin Nutr 2005;81:746-50.

26. Hamrick M, Pennington C, Webb $\mathrm{C}$ et al. Resistance to body fat gain in 'doublemuscled' mice fed a high-fat diet. Int J Obes 2006;30:868-70.

27. Gutin B. Child obesity can be reduced with vigorous activity rather than restriction of energy intake. Obesity 2008;16:2193-6.

28. Luu Y, Capilla E, Rosen C et al. Mechanical stimulation of mesenchymal stem cell proliferation and differentiation promotes osteogenesis while preventing dietary induced obesity. J Bone Miner Res 2009; 24:50-61.

29. Seubsman S, Sleigh A. Change in mean height of Thai military recruits from 1972 through 2006. J Epidemiol 2009;19:196201.

30. Kriemler S, Zahner L, Puder J et al. Weight-bearing bones are more sensitive to physical exercise in boys than in girls during pre- and early puberty: a cross-sectional study. Osteoporos Int 2008;19:1749- 
58.

31. Veldhuis J, Roemmich J, Richmond E et al. Endocrine control of body composition in infancy, childhood, and puberty. Endocr Rev 2005;26:114-46.

32. Nicklas T, O'Neil C, Fulgoni V. The role of dairy in meeting the recommendations for shortfall nutrients in the American diet. $\mathrm{J}$ Am Coll Nutr 2009;28:73-81S.
33. Seeman E, Delmas P. Bone quality--the material and structural basis of bone strength and fragility. N Engl J Med 2006; 354:2250-61.

34. Holick M, Chen T. Vitamin D deficiency: a worldwide problem with health consequences. Am J Clin Nutr 2008;87:1080-6S.

35. Lofgren B, Stenevi-Lundgren S, Dencker M et al. The mode of school transportation in pre-pubertal children does not influence the accrual of bone mineral or the gain in bone size - two year prospective data from the paediatric osteoporosis preventive (POP) study. BMC Musculoskel Dis 2010;11:2.

36. Gutin B. Diet vs exercise for the prevention of pediatric obesity: the role of exercise. Int J Obes, 2011;35:29-32. 